\title{
THE CAUCHY INTEGRAL FOR DIFFERENTIAL FORMS
}

\section{BY WALTER KOPPELMAN ${ }^{1}$}

Communicated by Murray Gerstenhaber, March 17, 1967

In a previous note, we introduced a certain determinant of differential forms (cf. formula (1.3) of [3]), which led to an elementary proof of the Cauchy integral formula for holomorphic functions of several complex variables. We now propose to amplify this procedure in order to obtain some integral representations for exterior differential forms.

We shall be considering certain mappings $\psi(\zeta, z)$ and $f(\zeta, z)$ of an open set $V \subset C^{n} \times C^{n}$ into $C^{n}$, where $\psi$ is of class $C^{\infty}$ and $f$ is holomorphic. Writing $\psi=\left(\psi_{1}, \cdots, \psi_{n}\right)$ and $f=\left(f_{1}, \cdots, f_{n}\right)$, we set $\langle\psi, f\rangle=\psi_{1} f_{1}+\cdots+\psi_{n} f_{n}$. Henceforth, we shall always assume that $\langle\psi, f\rangle \neq 0$ at the points under consideration. Now, instead of merely considering a single smooth mapping $\psi$, we take $n$ such mappings, $\psi^{(1)}, \psi^{(2)}, \cdots, \psi^{(n)}$. Each of these will be regarded as a column in terms of its components. We shall, furthermore, use the vectorvalued differential forms $\bar{\partial}_{\zeta} \psi=\sum_{j=1}^{n} \psi_{\bar{\zeta}_{j}} d \bar{\zeta}_{j}$ and $\bar{\partial}_{z} \psi=\sum_{j=1}^{n} \psi_{\bar{z}_{j}} d \bar{z}_{j}$. With this notation, we look at the $n \times n$ determinant

$$
\begin{gathered}
j=2, \cdots, \mu \quad k=\mu+1, \cdots, n \\
D\left(\left\langle\psi^{(1)}, f\right\rangle^{-1} \psi^{(1)}, \bar{\partial}_{z}\left(\left\langle\psi^{(j)}, f\right\rangle^{-1} \psi^{(j)}\right), \bar{\partial}_{\zeta}\left(\left\langle\psi^{(k)}, f\right\rangle^{-1} \psi^{(k)}\right)\right),
\end{gathered}
$$

which will be viewed as a double form on $V$ (cf. [4, p. 35]). As in [3], we can now state

Proposition 1. The expression (1) is independent of the choice of the mapping $\psi^{(1)}$.

Along the same direction, we also have

Proposition 2.

$$
\begin{gathered}
j=2, \cdots, \mu \quad k=\mu+1, \cdots, n \\
\text { (i) } \quad D\left(\bar{\partial}_{z}\left(\left\langle\psi^{(1)}, f\right\rangle^{-1} \psi^{(1)}\right), \bar{\partial}_{z}\left(\left\langle\psi^{(j)}, f\right\rangle^{-1} \psi^{(j)}\right), \bar{\partial}_{\zeta}\left(\left\langle\psi^{(k)}, f\right\rangle^{-1} \psi^{(k)}\right)\right)=0 ; \\
j=2, \cdots, \mu \quad k=\mu+1, \cdots, n \\
\text { (ii) } \quad D\left(\bar{\partial}_{\zeta}\left(\left\langle\psi^{(1)}, f\right\rangle^{-1} \psi^{(1)}\right), \bar{\partial}_{z}\left(\left\langle\psi^{(j)}, f\right\rangle^{-1} \psi^{(j)}\right), \bar{\partial}_{\zeta}\left(\left\langle\psi^{(k)}, f\right\rangle^{-1} \psi^{(k)}\right)\right)=0 .
\end{gathered}
$$

With the aid of Propositions 1 and 2, we can derive

1 This research has been supported by the National Science Foundation under grant GP 6102. 
Proposition 3.

$$
\begin{aligned}
& (n-q) \bar{\partial}_{\zeta} D\left(\langle\psi, f\rangle^{-1} \psi, \bar{\partial}_{z}\left(\langle\psi, f\rangle^{-1} \psi\right), \bar{\partial}_{\zeta}\left(\langle\psi, f\rangle^{-1} \psi\right)\right) \\
& \text { and } \quad \\
& =(-1)^{q-1} q \bar{\partial}_{z} D\left(\langle\psi, f\rangle^{-1} \psi, \bar{\partial}_{z}\left(\langle\psi, f\rangle^{-1} \psi\right), \bar{\partial}_{\zeta}\left(\langle\psi, f\rangle^{-1} \psi\right)\right)
\end{aligned}
$$

Proposition 4. The difference of two forms

$$
\begin{array}{cc}
q & n-q-1 \\
D\left(\left\langle\psi^{(j)}, f\right\rangle^{-1} \psi^{(j)},\right. & \bar{\partial}_{z}\left(\left\langle\psi^{(j)}, f\right\rangle^{-1} \psi^{(j)}\right), \\
\left.\bar{\partial}_{\zeta}\left(\left\langle\psi^{(i)}, f\right\rangle^{-1} \psi\right)\right), & j=1,2,
\end{array}
$$

is equal to a sum of $\bar{\partial}_{\zeta}$-exact and $\bar{\partial}_{z}$-exact terms.

We shall now state a formula for exterior differential forms of type $(0, q)$, which is analogous to the classical formula of Martinelli and Bochner for holomorphic functions. For this, we set

$$
\begin{aligned}
& \text { (2) } u_{n, q}(\zeta, z)=(-1)^{q}\left(\begin{array}{c}
n-1 \\
q
\end{array}\right) D\left(\langle\bar{\zeta}-\bar{z}, \zeta-z\rangle^{-1}(\bar{\zeta}-\bar{z})\right. \text {, } \\
& q \quad n-q-1 \\
& \left.\bar{\partial}_{z}\left(\langle\bar{\zeta}-\bar{z}, \zeta-z\rangle^{-1}(\bar{\zeta}-\bar{z})\right), \bar{\partial}_{\zeta}\left(\langle\bar{\zeta}-\bar{z}, \zeta-z\rangle^{-1}(\bar{\zeta}-\bar{z})\right)\right), \\
& 0 \leqq q \leqq n-1, \\
& u_{n,-1}(\zeta, z)=u_{n, n}(\zeta, z) \equiv 0,
\end{aligned}
$$

and

$$
\omega(f)=d_{5} f_{1} \wedge \cdots \wedge d_{\xi} f_{n}
$$

Let us assume that $D$ is a relatively compact domain in $C^{n}$, with a piecewise smooth boundary $\partial D$ consisting of a finite number of connected components. If $\gamma$ is a (smooth) differential form of type $(0, q)$ on $\bar{D}$, the closure of $\mathscr{D}$, we have

Theorem 5.

$$
\begin{aligned}
& \frac{(-1)^{n(n-1) / 2}}{(2 \pi i)^{n}}\{ \int_{\partial \mathscr{D}} \gamma(\zeta) \wedge u_{n, q}(\zeta, z) \wedge \omega(\zeta) \\
&-\int_{\mathscr{D}} \bar{\partial} \gamma(\zeta) \wedge u_{n, q}(\zeta, z) \wedge \omega(\zeta) \\
&\left.-\bar{\partial}_{z} \int_{\mathscr{D}} \gamma(\zeta) \wedge u_{n, q-1}(\zeta, z) \wedge \omega(\zeta)\right\}=\gamma(z), \quad \text { if } z \in \mathscr{D} \\
&=0, \quad \text { if } z \in \bar{D} .
\end{aligned}
$$


With our notation, this formula is valid for $0 \leqq q \leqq n$. Observe that when $\gamma$ is $\bar{\partial}$-closed, its $\bar{\partial}$-cohomology class is essentially governed by the boundary term of the left-hand side.

Using this formula in conjunction with Proposition 3, we readily obtain a local version of an extension theorem treated by Andreotti and Vesentini [1] and employed by us in $\$ 4$ of our note [3]. If we apply Proposition 4 to this formula, we obtain a "vanishing theorem," which we now state. Let us say that an algebra $a$ (over $C$ ) of $C^{\infty}$ functions, defined in a domain in $C^{n}$, is of antiholomorphic rank $r-1$, if $h_{1}, \cdots, h_{r} \in Q \Rightarrow \bar{\partial} h_{1} \wedge \cdots \wedge \bar{\partial} h_{r} \equiv 0$, and if there exist functions $g_{1}, \cdots, g_{r-1} \in Q$, with $\bar{\partial} g_{1} \wedge \cdots \wedge \bar{\partial} g_{r-1} \not \equiv 0$. If $K$ is a compact subset of the domain, the restriction of $a$ to $K$ will be denoted by $a \mid K$. This algebra $a \mid K$ becomes a normed algebra under the supremum norm relative to $K$. As usual, we denote the sheaf of germs of holomorphic functions by $\mathcal{O}$.

THEOREM 6. Suppose that $D$ is a domain in $\mathbf{C}^{n}$, with the property that, for each compact set $K \subset D$, there exists an algebra $Q(K)$ of $C^{\infty}$ functions on $D$, containing $1, z_{1}, \cdots, z_{n}$, and of antiholomorphic rank $\leqq r-1$, such that the joint spectrum $\hat{K}$ of $z_{1}, \cdots, z_{n} \in Q(K) \mid K$ is contained in $D$; then $H^{q}(D, \mathcal{O})=0, q \geqq r$.

The connection between this theorem and H. Cartan's Theorem B for the structure sheaf $\mathcal{O}$ over a domain of holomorphy $D$ is provided by Theorem 4 of Bishop's paper [2].

\section{BIBLIOGRAPHY}

1. A. Andreotti and E. Vesentini, Carleman estimates for the Laplace-Beltrami equation on complex manifolds, Publ. Math. I. H. E. S. no. 25 (1965), 81-130.

2. E. Bishop, Some global problems in the theory of functions of several complex variables, Amer J. Math. 83 (1961), 479-498.

3. W. Koppelman, The Cauchy integral for functions of several complex variables, Bull. Amer. Math. Soc. 73 (1967), 373-377.

4. G. de Rham, Variêtés différentiables, Hermann, Paris, 1960.

The Institute FOR AdVANCED STUDY AND

The University of Pennsylvania 